\title{
IV. Krise und Wende: Die Pariser Außenministerkonferenz 1946
}

Die Pariser Außenministerkonferenz, obwohl nicht vorrangig mit der deutschen Frage befaßt, war die entscheidende Zäsur in der Geschichte des Kontrollrats: An ihrem Ende war der Versuch erkennbar gescheitert, die angestrebte Wirtschaftseinheit über die Wahrung bzw. Wiederherstellung der politischen Einheit zu gewährleisten. Trotz mancher Einzelerfolge und Kompromisse war kein Einvernehmen über eine europäische Friedensordnung erzielt worden, die den Rahmen zur Lösung der Deutschlandfrage geboten hätte. Die in Paris durchscheinende Verknüpfung der europäischen, ja globalen Entfremdung mit der besatzungspolitischen Enttäuschung in Deutschland bereitete das Klima für einen Meinungsumschwung, der in Großbritannien seit geraumer Zeit unter dem Aspekt einer „Revision von Potsdam“ diskutiert wurde. Der Alltag des Friedens hatte die Hoffnungen, die Illusionen von Potsdam eingeholt. Die Zwänge der Besatzungspraxis forderten die Revision der deutschlandpolitischen Entwürfe; die Bindungen des Einstimmigkeitsprinzips im Kontrollrat begründeten den Wunsch nach erweiterter Handlungsfreiheit, die nur um den Preis der Aufkündigung des prekär gewordenen Konsenses zwischen den Alliierten zu haben war. Kurz: Es mußten Entscheidungen getroffen werden.

Stalins Rede vom 9. Februar, Kennans „langes Telegramm“ vom 22. Februar und Churchills Fulton-Rede vom 5. März 1946 markierten, auch wenn letztere sich als Reaktion auf Stalins Äußerungen verstanden, den synchronen Takt dieses Entfremdungsprozesses. Das Unbehagen am Besatzungsalltag in Deutschland gab jedoch in erster Linie nicht den Militärregierungen Anlaß zu Revisionsbestrebungen, auch nicht den verantwortlichen Ministern, sondern es waren die bürokratischen Apparate in den Hauptstädten, die die Initiative ergriffen. Die Militärregierungen hielten mehrheitlich Kompromisse zur effizienteren Ausgestaltung des Kontrollapparats auf pragmatischer Ebene noch für möglich. Indem sie die Situation „offen“ zu halten suchten, wollten sie die Behandlung Deutschlands als Einheit zumindest nicht ausschließen. Ihre Klagen über die besatzungspraktischen Schwierigkeiten in Deutschland verstärkten jedoch den Reorientierungsprozeß der Planungsbürokratien, die sich mehr an übergreifenden strategischen Interessen orientierten und weniger auf pragmatische als auf prinzipielle Regelungen bedacht waren ${ }^{1}$. Die Militärgouverneure waren immer seltener in der Lage, die Entscheidungsprozesse auf den höchsten politischen Ebenen zu beeinflussen; Kompromisse, in Berlin ausgehandelt, waren in den Hauptstädten bzw. auf den Außenministerkonferenzen nicht mehr erwünscht. Die daraus erwachsende Handlungsunfähigkeit des Kontrollrats verstärkte die Neigung, Entscheidungen weiter hinauszuzögern. Die Einheit Deutschlands bzw. die Einheit der Alliierten in Deutschland war bereits auf der Pariser Außenministerkonferenz ohnehin nicht mehr

1 NA, RG 59/OWEA/Misc. German Files, box 2, folder: Murphy (DeWitt C. Poole, Murphy, Harold Deutsch; Februar bis April 1946). 
die primäre Option. Durch Vertagung der Deutschlandfrage auf die nächste Ratssitzung, die vordergründig mit der Verzögerung des Friedensschlusses mit den früheren Verbündeten des Reiches begründet werden konnte, sollte Zeit für eine grundsätzliche Neubestimmung der jeweiligen nationalen Deutschlandpolitik gewinnen.

\section{1. „Revision von Potsdam“? Die Reorientierung der amerikanischen und britischen Deutschlandpolitik im Sommer 1946}

In den USA war die Debatte über eine Neudefinition der Deutschlandpolitik im Dezember 1945 durch die angestrebte Übergabe der Besatzungsverwaltung vom War an das State Department ausgelöst worden. Clay hatte die Direktive JCS 1067 in ihrer durch Potsdam veränderten Form als „eine, mit geringen Ausnahmen, brauchbare Politik" bezeichnet; nicht sie, sondern die französische Blockade sei das Haupthindernis für eine effektivere Besatzungspolitik, die das State Department vor allem im wirtschaftlichen Bereich gefordert hatte ${ }^{2}$. Die Stagnation im Kontrollrat und auf der Moskauer Außenministerkonferenz hatte im Dezember 1945 und Januar 1946 Clay und das Kriegsministerium zu „ernsthaften Überlegungen“ veranlaßt. Letzteres hatte dem stellvertretenden Militärgouverneur im Bedarfsfalle eine „Modifikation oder Ausweitung“ durch Einzelmaßnahmen und „Ergänzungen“ gestattet. Diesem erschien, mehr noch als seinen Vorgesetzten, eine Revision der realen Politik, aber keine offizielle Generalrevision der Direktive erforderlich bzw. erstrebenswert. Doch die Hoffnung, eine Grundsatzdebatte aus innen- wie außenpolitischen Gründen zu vermeiden, zerschlug sich; diese hatte längst begonnen. Nachdem Marineminister Forrestal und Acheson Kennans „langes Telegramm“ zum Anlaß für eine grundsätzliche Neubestimmung der amerikanischen Außenpolitik und des Verhältnisses zur Sowjetunion genommen hatten, hatte das State-War-Navy Coordinating Committee bereits im März 1946 eine Überprüfung aller Europa betreffenden Direktiven angeordnet ${ }^{3}$. Und Clay, der Kennans „langes Telegramm“ mit großer Verärgerung zur Kenntnis genommen hatte, lieferte durch sein ungeduldiges Drängen und seine verärgerten Schuldzuweisungen im Kontrollrat dazu selbst die Argumente ${ }^{4}$.

Clay und Murphy in Berlin ${ }^{5}$, Kennan und Smith in Moskau ${ }^{6}$, Harris, Matthews und Riddleberger in Washington ${ }^{7}$ sahen die USA in Deutschland von der zielstrebigen Politik der Kommunisten in die Defensive gedrängt. Einigkeit bestand in der Beschreibung der Ausgangslage: Das Warten auf ein Einlenken Frankreichs in der Frage der Zentralverwaltungen wurde der akuten Lage nicht mehr gerecht und hinderte die Anglo-Amerikaner an einem konstruktiven Neuaufbau, während die Sowjetunion den Kommunisten in der SBZ die Machtpositionen übertrug und in den Westzonen durch ihre Einheitspropaganda um Sympathien warb. Der durch die sowjetische Poli-

2 CP, S. 132 ff. (10.12. 1945), 147 (4. 1. 1946).

3 NA, RG 59, 740.00119 Control(Germany)/3-1846.

4 Mai, Globalstrategie, S. 64. Vgl. zu Clay oben S. $66 \mathrm{f}$.

5 CP, S. 165 f. (22. 1. 1946). FRUS, 1946/V, S. 505 ff. (Murphy, 24. 3. 1946).

6 FRUS, 1946/V, S. 516 ff. (Kennan, 6.3. 1946), 535 f. (Smith, 2. 4. 1946).

7 NA, RG 59, 740.00119 Control(Germany)/3-2646 (Harris), /3-2646 (Matthews), /4-1546 (Mendershausen); RG 59/ASSOA, box 2 (26. 3. 1946); RG 59/CED, box 2, folder: G 710 (Riddleberger, 28. 3. 1946). 
tik im Osten ausgelöste „Trend zur Teilung“ eröffnete aber, so Kennan, die Chance für den Versuch, „die westlichen Zonen Deutschlands zu retten, indem man eine Mauer gegen die östliche Penetration errichtet und sie in den internationalen Rahmen Westeuropas integriert statt in ein vereintes Deutschland“. Dagegen schien Harris der sicherste Weg, Westdeutschland für den Westen zu verlieren, daß man „dem Kommunismus den Weg durch die Teilung Deutschlands“ zu verbauen suche. Ähnlich differierten die Ansichten in der Zentralverwaltungsfrage. Kennan teilte die französischen Vorbehalte gegenüber einer Zentralisierung als möglichem sowjetischen Einfallstor, während Harris, Clay und Murphy in einer Art Magnet-Theorie die Zentralinstanzen als Voraussetzung für einen wirtschaftlichen und politischen Wiederaufschwung erachteten, der ganz Deutschland für den Westen gewinnen werde. In jedem Fall aber, das war Konsens, sahen sich die USA angesichts der ihnen „aufgezwungenen“ Wahl zwischen Teilung und Einheit gefordert, ein „konstruktives“ deutschlandpolitisches Konzept zu entwickeln. Die von Harris ins Auge gefaßte Errichtung der Zentralverwaltungen bzw. die von ihm für unabdingbar gehaltene „Wiedererrichtung einer deutschen Zentralregierung" einerseits, die von Riddleberger angedeutete Option für eine Westlösung andererseits verlangten jedoch mehr als die punktuelle Revision des Potsdamer Abkommens und der Direktive JCS 1067, soweit diese sich als „unzuträglich oder unangemessen“ erwiesen und Maßnahmen im Kontrollrat oder in der amerikanischen Zone behindert hatten. Die Konzeption für eine langfristige deutschlandpolitische Strategie wurde eingefordert, dazu ein effektives Wirtschaftsprogramm, das die Revision des Industrieniveauplans erforderlich machen würde.

Ergebnis des internen Diskussionsprozesses war ein weiteres Papier von Harris, das mit Hilldring abgestimmt war und über Riddleberger Eingang in die Delegation auf der Pariser Außenministerkonferenz fand. Darin wiederholte er zunächst seine Thesen: Nach einem Jahr der Erfahrungen sei es an der Zeit, die Grundannahmen von JCS 1067 zu überprüfen; zudem brauche OMGUS für den Fall „andauernder Teilung“ eine „Propagandawaffe“. „Solange es ungefähr siebzig Millionen Deutsche im Herzen Europas gibt“, spitzte er dann jedoch zu, „ist es von lebenswichtiger Bedeutung für uns, daß diese Deutschen in einer Weise handeln und denken, die mit unseren Interessen vereinbar ist. Man kann nicht davon ausgehen, daß angesichts des chaotischen Zustands ihres Landes sie so denken und handeln werden, wenn wir sie nicht in dieser Richtung zu beeinflussen suchen. Wir befinden uns daher im Wettbewerb mit der Sowjetunion um die Seele des deutschen Volkes, und, ob wir es wollen oder nicht, wir müssen ihnen so viel bieten, wie wir ohne Schaden oder Gefahr für uns selbst können.“ Da die nationale Einheit das wichtigste Problem der deutschen „Seele“ sei, werde „die Besatzungsmacht den größten Einfluß ausüben [...], die am deutlichsten als Protagonist dieser Politik auftritt“. Er forderte den Wettbewerb mit der Sowjetunion um das „Menschenmaterial, das aus verschiedenen Gründen in die NSDAP ging“, also eine veränderte Entnazifizierungspraxis, dazu die baldige Propagierung einer gesamtdeutschen Regierung als zweite Stufe nach der Errichtung deutscher Zentralverwaltungen; ohne festen Zeitplan, aber doch bald: „Die kommunistische Partei ist bereits an der Arbeit."

${ }^{8}$ NA, RG 59, 740.00119 Control(Germany)/4-2046 und /5-246 (Explanatory Comment on the Draft Directive, 1.5. 1946). 
Eine gegenteilige Position bezog Perry Laukhuff, die Murphy zwar nicht teilte, aber doch für wichtig genug hielt, um sie seinerseits an die amerikanische Delegation in Paris weiterzuleiten: Die sowjetische Position in Deutschland sei derart stark, daß es sinnlos sei, sie ausgerechnet dort herauszufordern. Die USA müßten in Deutschland ein Gegengewicht schaffen, indem sie einen „eigenen Eisernen Vorhang“ herunterließen und die SBZ durch einen Vorstoß in Österreich, Polen und der Tschechoslowakei zu „umfassen“ suchten. Die Zentralverwaltungen sah er als „zweischneidiges Schwert“, da die Sowjets solche als arbeitsfähiges Instrument nur akzeptieren würden, wenn die SED in ihnen über eine starke Stellung verfügte. Er befürwortete als demonstrativen, die Öffentlichkeit wachrüttelnden Schritt den Rückzug aus dem Kontrollrat und die Teilung Deutschlands. Allein durch einen brutalen Schnitt - auch wenn das bedeute, die SBZ „für einige Zeit abzuschreiben“ - werde langfristig eine sowjetische Penetration „durch den weichsten Punkt der Linie, die jetzt Europa teilt“, zu verhindern sein, „weil wir unsicher über unsere Politik und zur Zeit in uns selbst gespalten sind und unfähig, frei zu handeln, inmitten eines feindlichen Volkes"c? Wenige Tage später ging Kennan noch einen Schritt weiter, als er forderte, die USA sollten das Potsdamer Abkommen aufkündigen. Die Hinnahme der Oder-Neiße-Linie sei ein Fehler gewesen, der den Franzosen Berechtigung gebe, Gleiches im Westen zu verlangen; versteckt hinter dem französischen Veto könnten sich die Sowjetunion bzw. die Kommunisten zum Protagonisten der deutschen Einheit machen. Eine Aufkündigung des Potsdamer Abkommens durch die USA, wofür „reichlich Rechtfertigung“ bestünde, würde die Sowjets unter Zugzwang setzen: Entweder müßten sie die polnischen Kommunisten desavouieren, wenn sie den Deutschen die Gebiete östlich von Oder und Neiße anböten, oder im umgekehrten Falle ihre nationale Propaganda in Deutschland diskreditieren; „und wir wären dann frei, mit der Organisation Westdeutschlands voranzuschreiten, unabhängig von den Russen, ohne als Gegner eines vereinten Deutschlands angeprangert zu werden." ${ }^{10}$

Angesichts des enttäuschenden Verlaufs des ersten Konferenzabschnitts war die Hoffnung, durch Vertagung oder Lockung der Grundsatzentscheidung ausweichen zu können, erneut schwächer geworden. Jetzt wurde „zum ersten Mal die Möglichkeit eines Zusammenbruchs der Vier-Mächte-Kontrolle ernsthaft erwogen [...], obwohl jede Anstrengung von den westlichen Mächten unternommen werden wird, um das abzuwenden, was allgemein als katastrophale Entwicklung betrachtet wird. [...] Die VierMächte-Kontrolle Deutschlands ist daher durch gegenseitiges Mißtrauen gefährdet sowie [durch] die fehlende Bereitschaft, gewisse unmittelbare Vorteile der uneingeschränkten zonalen Jurisdiktion aufzugeben. Wenn dieser Stillstand anhält, dann besteht die Möglichkeit, daß auf die Vereinigung der drei Westzonen zurückgegriffen werden muß, damit die sowjetisch-westliche Teilung Deutschlands verfestigend. Das könnte auf die Integration Westdeutschlands in eine westeuropäische Gruppierung hinzielen, wenn Europa in rivalisierende Gebiete zerfällt. In jedem Fall ist Deutschland jetzt zum wichtigsten Schauplatz gegensätzlicher Ideologien und politischer Konflikte zwischen den Sowjets und der anglo-amerikanischen Gruppe geworden.“

9 NA, RG 59, 740.00119 Control(Germany)/5-346 (Appraisal of Quadripartite Control and Soviet Policy, 25. 4. 1946).

10 NA, RG 59, 740.00119 Control(Germany)/5-1046 (Russian Policy in Germany). 
Die Deutschlandfrage hatte ein „kritisches Stadium“ erreicht, und die Entscheidung über Einheit oder Teilung wurde für die „nahe Zukunft“ erwartet ${ }^{11}$.

Das war nicht die Entscheidung zur Teilung. Aber die axiomatische Erwartung des Scheiterns mußte hemmend auf den Willen zur Einheit wirken: Das Mißtrauen war zu groß geworden, das Sicherheitsdenken zu dominierend, als daß der politische Mut und die geistige Bereitschaft für den entscheidenden Durchbruch noch denkbar gewesen wären. Aber noch war Byrnes nicht bereit, dem Drängen seiner Mitarbeiter, und keineswegs nur der „hard-liner“, nachzugeben. In der zweiten Konferenzphase sollte erneut ein Versuch unternommen werden, die Sowjetunion durch neue Angebote zur Kooperation zu bewegen - ja, notfalls zu zwingen. Zu diesem Zweck wurde ein Bündel von Maßnahmen bzw. Vorschlägen ausgearbeitet, das - darin lag bereits ein neuer Vorbehalt - als „letzter Test“ dienen sollte, um im Falle eines Scheiterns dieses Vorstoßes und damit auch des Kontrollrats „die Schuld für den Bruch von Potsdam den Sowjets anheften“ zu können. Die Blockade des Kontrollrats sollte aufgebrochen werden, indem der Sowjetunion das Aussetzen des Demontagestopps für 60 bis 90 Tage angeboten wurde, wenn diese im Gegenzug sich zu Verhandlungen über ein gemeinsames Export-Import-Programm verpflichtete und wenn die Ausführung der gemeinsamen Politik, „bis zur Errichtung voll entwickelter deutscher Zentralverwaltungen, Alliierten Büros der vier Mächte übertragen wird, die von Stäben deutscher technischer Experten unterstützt werden“. Waren Fortschritte zur Herstellung der wirtschaftlichen Einheit nicht zu erzielen, würde das Reparationsprogramm am Ende dieser Phase ganz eingestellt werden. Clay unterstützte diese Strategie im Kontrollrat: Die Behandlung Deutschlands als Wirtschaftseinheit sei nur durch die Errichtung von deutschen Zentralverwaltungen zu erzielen. „Wenn diese Verwaltungen nicht erreicht werden können und/oder die Grenzen des besetzten Deutschlands verändert werden sollen, wird die gegenwärtige Konzeption von Potsdam bedeutungslos.“ Die Verantwortung für diese Stagnation wies er - wie im Bereich von Ruhrfrage, Rheinland-Abtrennung, Reparationen und politischer Verfassung eines föderierten Deutschlands in erster Linie Frankreich zu. Der Ausweg aus dieser Krise, die „Verschmelzung“ der britischen und amerikanischen Zone, verlangte indes die Preisgabe bzw. Revision der bisherigen Deutschlandpolitik ${ }^{12}$. Eine solche Entscheidung mußte durch den Versuch flankiert werden, die Widerstände Frankreichs gegen gesamtdeutsche Regelungen zu überwinden: um einmal den Sowjets die Gelegenheit zu nehmen, sich hinter dem französischen Veto zu „verstecken“, und um zum anderen die Voraussetzungen für eine trizonale Westlösung zu schaffen.

Ziel des doppelten Vorstoßes in Paris und Berlin war ein frühzeitiger Friedensvertrag mit Deutschland bzw. die Festlegung von relativ verbindlichen Prinzipien für denselben, um angesichts der Bemühungen, in der SBZ vollendete Tatsachen zu schaffen, zukünftig die innerdeutsche Entwicklung sowjetischem Einfluß zu entziehen ${ }^{13}$. Ein Rückzug der Sowjetunion aus Deutschland schien nur zu erreichen, wenn ihr (wie Frankreich auch) die dauerhafte Entmilitarisierung Deutschlands zugesichert

11 NA, RG 59/CED, box 3, folder: Current Political Developments (15. 5. und 1. 6. 1946).

12 FRUS, 1946/V, S. 549 ff. (Acheson an Byrnes, 9. 5. 1946). NA, RG 59/CED, box 2, folder: G 710 (7. 6. 1946). CP, S. 212 ff. (26. 5. 1946), 221 f. (27. 5. 1946). BA, Z 45 F/OMGUS, POLAD/745/27 (Murphy an Clay, 10. 5. 1946). AMAE, Y 391, Bl. $200 \mathrm{ff}$. (Murphy).

13 NA, RG 59, 740.00119 Control(Germany)/4-2446 (Murphy an Byrnes). Vgl. FRUS, 1946/II, S. 400 ff. (15. 5. 1946), 428 (16. 5. 1946). 
wurde. Das gab dem in seinen Grundzügen bereits 1945 entwickelten Byrnes-Plan neue Bedeutung, durch eine amerikanische Garantie die Entwaffnung und Kontrolle Deutschlands für 25 Jahre zu gewährleisten - d. h. durch Abkehr von den bisherigen Prinzipien der amerikanischen Außenpolitik und Übernahme eines langfristigen Engagements in Deutschland und Europa ${ }^{14}$. Aber eben diese Einbettung in einen gesamteuropäischen Rahmen machte den Plan für die Sowjetunion uninteressant, bedeutete das doch, daß sie die Realisierung ihrer Ansprüche gegenüber Deutschland mit dem langfristigen Engagement der USA in Mitteleuropa erkaufen mußte ${ }^{15}$. Daher stand Mitte Mai, am Ende der ersten Konferenzphase in Paris, fest, daß die Sowjetunion den „Test“ nicht bestanden hatte. Die Forderung nach einem neuen, „positiven" deutschlandpolitischen Ansatz wurde immer lauter, der die Blockade der VierMächte-Verwaltung aufbrach, die Deutschen auf die Seite der USA und des Westens zog, eine Lösung für die Ruhrfrage eröffnete (notfalls im Rahmen einer Bizone) und dem OMGUS-Personal eine Perspektive für die tagespolitische Arbeit vermittelte ${ }^{16}$.

Angesichts der festgefahrenen Fronten war wenig Gutes für den zweiten Abschnitt der Pariser Konferenz zu erwarten, der am 15. Juni begann. Bereits jetzt war zu erkennen, daß die Entwicklung immer stärker auf die Bildung einer Bizone hinauslief und die deutsche Frage immer intensiver als Teil nur noch der westeuropäischen Rekonstruktionsproblematik begriffen wurde. Matthews schlug seinem Außenminister vor, sollte ein neuerlicher Vorstoß scheitern, ,die ernsthafte Hoffnung, zumindest vorübergehend, auf die Wiederherstellung der Einheit Deutschlands“ aufzugeben und auf eine $\mathrm{Bi}$ - oder Trizone im Westen hinzuarbeiten. Dabei „wäre es klüger, nur mit den Briten zusammenzugehen als einen unmöglichen Preis für die französische Teilnahme zu zahlen“. „Während wir versuchen, einen Teil Deutschlands zu organisieren, wäre es klug, weiterhin auf eine sofortige Friedensregelung für Deutschland als Ganzes zu drängen und unsere Teilnahme am Alliierten Kontrollrat in Berlin fortzusetzen. Wenn es sich als zweckmäßig erweist, sollten unsere militärischen Regierungsbehörden alle Politikentscheidungen einer Vier-Mächte-Diskussion unterbreiten und dann“, das war der erneute Rückgriff auf die amerikanische Interpretation des Kontrollabkommens seit dem Frühjahr 1945, „nach einem angemessenen Zeitraum das Nichtzustandekommen von Vereinbarungen feststellen und die notwendigen Vorkehrungen für eine bilaterale oder trilaterale Kooperation treffen. “17 Hilldring unterstützte diese Position, hinter der „Fassade“ des Kontrollrats auf eine Westlösung zuzusteuern.

14 Kreikamp, Deutschlandpolitik. FRUS, 1946/II, S. 146 f., 190 ff. Der Plan ging auf Vorschläge von Senator Vandenberg vom 10.1. 1945 zurück. Ein Entwurf vom 17. 2. 1945 in: FRUS, 1945/III, S. 424 ff. Erste Vorüberlegungen des State Department in: FRUS, Potsdam I, S. 450 ff. Byrnes kündigte den Plan im Oktober bzw. Dezember 1945 Bidault und Molotow als Ende des Isolationismus an. Byrnes, Speaking, S. 171, 176. AMAE, Y 134, Bl. 130. Briten und Franzosen verstanden den Plan als prinzipielle Neuorientierung der Außenpolitik der USA. PRO, FO 371/55842/C4800 (2. 5. 1946). AN, 457 (Bidault) AP 7 (14.3. 1947). Daß Byrnes den Plan ohne Information des Präsidenten durch Riddleberger hatte entwickeln lassen, war ein Grund für die Entfremdung zwischen ihm und Truman. TL, Oral History Collection, Riddleberger (1972), S. 86. Den entsprechenden Passus seiner Stuttgarter Rede ließ Byrnes sich von Truman genehmigen. Clay, Entscheidung, S. 97.

$15 \mathrm{Vgl}$. unten S. $165 \mathrm{f}$.

16 CP, S. 217 (26. 5. 1946), 226 ff. (2.6. 1946). FRUS, 1946/II, S. 486 ff. (Patterson an Byrnes, 11. 6. 1946). NA, RG 59/CED, box 3, folder: Current Political Developments (1.6. 1946, S. 3); RG 59, 740.00119 Control(Germany)/6-2646; RG 59/ASSOA, box 1 (10.6. 1946).

17 NA, RG 59/CED, box 2, folder: G 710 (Matthews an Byrnes, 7. 6. 1946). AMAE, Y 286, Bl. 57 (Bonnet, 11.6. 1946). 
Hauptaufgabe der amerikanischen Politik müsse die Integration der deutschen Wirtschaft in eine europäische Ökonomie sein. Die Voraussetzungen dafür seien zunächst auf Vier-Mächte-Ebene zu suchen; doch auch er rechnete nicht mehr damit, daß eine solche Einigung im Kontrollrat möglich sei ${ }^{18}$. Zwar sei durch Zurückhaltung und Flexibilität bei der Organisation der bizonalen Kooperation der Weg für den Anschluß der SBZ offenzuhalten; doch wenn die Sowjetunion erwartungsgemäß einen solchen Schritt ablehnte, sei die Teilung unvermeidlich ${ }^{19}$. Schon gab es Vorschläge, auf einer speziellen Außenministerkonferenz zur Deutschlandfrage prüfen zu lassen, wie weit die Potsdamer Beschlüsse realisiert worden seien, um derart eine Legitimation für die unilaterale Revision der amerikanischen Politik zu gewinnen ${ }^{20}$. Dem standen nur noch vereinzelt Gegenstimmen gegenüber, so etwa die Empfehlung des „Litchfield Committee", Deutschland unter UN-Treuhandschaft zu stellen, in der Hoffnung, daß durch die größere Distanz aller Besatzungsmächte „größere Einheit“ zu erreichen sei als im Kontrollrat oder auf den Außenministerkonferenzen ${ }^{21}$. Die USA sahen sich vor der Entscheidung, ob sie einen neuen Kompromiß suchen und sich auf die endlose Konferenzdiplomatie einlassen oder den Bruch herbeiführen sollten.

Nach französischem Eindruck war der Richtungsstreit innerhalb der amerikanischen Delegation bis Konferenzbeginn nicht entschieden; aber ein Erfolg der immer schwächer werdenden kompromißbereiten Fraktion setzte sowjetische Zugeständnisse voraus $^{22}$. Wenn sich die USA bis dahin noch nicht definitiv entschieden hatten, so war dies im wesentlichen auf das Zögern von Außenminister Byrnes zurückzuführen. Dieser hatte sich des Drucks Clays, Murphys und seiner anderen Berater erwehren können, solche weitreichenden Entscheidungen zu treffen, ehe nicht die Chancen für eine pragmatische Einigung mit der Sowjetunion abschließend ausgelotet waren. Es war aufschlußreich für die fließenden Fronten, daß noch Mitte Juli weder seinen Mitarbeitern noch den Briten klar war, ob das Angebot der interzonalen Kooperation sich de facto nur an die britische Zone richtete oder auch an die Sowjetunion (und Frankreich) ${ }^{23}$. Nach dem Scheitern der Pariser Konferenz konnte es jedoch nur noch darum gehen, durch geschicktes Taktieren die Ausgangsposition für eine immer wahrscheinlicher bzw. notwendiger werdende Westlösung zu verbessern und den Sowjets die Verantwortung für die Teilung zuzuschieben. Byrnes, der seit längerem in Kongreß und Öffentlichkeit wegen seiner „weichen“ Politik unter Druck stand, blieb nichts anders mehr übrig, als dem Drängen der Mehrzahl seiner Mitarbeiter wie des OMGUS nachzugeben und eine Kursrevision einzuleiten. Fast automatisch kamen damit die Vorstellungen zum Zuge, die Clay am 26. Mai entwickelt hatte und am 19. Juli erneut vortrug. Er, Draper und einige Beamte in Washington „waren absolut davon überzeugt, daß es keine Möglichkeit gab, die Beschlüsse von Potsdam in der Form auszuführen, wie wir es gehofft hatten“; für sie stand jetzt fest, „daß der Kontrollrat unfähig

18 NA, RG 59/ASSOA, box 1 (Hilldring an Harris, 11.6. 1946; Bezug: Harris, wie Anm. 8).

19 AMAE, Y 377, Bl. 19 f. (Botschaft Washington, 17. 7. 1946).

20 NA, RG 59/CED, box 2, folder: G 710 (Harris, 8. 7. 1946); RG 59, 740.00119 Control(Germany)/7146 (Claxton an Hilldring). PRO, FO 943/488 (Botschaft Washington, 6. 8. 1946).

21 BA, Z 45 F/OMGUS, POLAD-TS/33/1.

22 AMAE, Y 147, Bl. 18 f.; Y 286, Bl. 57.

23 NA, RG 59, 740.00119 Control(Germany)/6-1946 (Lightner an Riddleberger, 19.7. [sic] 1946). PRO, FO 371/55700 (Franklin, 23. 7. 1946). Robertson vermutete im Herbst 1946 aufgrund der Verhandlungen im Kontrollrat, die Sowjets würden sich bis zum Frühjahr entscheiden, „sich mit den übrigen Zonen zusammen in eine Verwaltung zu begeben“. AVBRD, Bd. 1, S. 885. 
sei, sich auf irgendwelche wichtigen Entscheidungen in Deutschland zu einigen". Auf der Grundlage dieser pessimistischen Einschätzung legten Byrnes, Riddleberger, Clay und Murphy in Paris die Grundzüge der Stuttgarter Rede fest ${ }^{24}$. Da es als immer drängender empfunden wurde, Molotows Pariser Statement vom 10. Juli zu konterkarieren, hielt Byrnes seine Grundsatzrede bereits am 9. September ${ }^{25}$, ohne die Ergebnisse eines am 31. Juli im State Department eingesetzten Deutschlandkomitees abzuwarten, das beauftragt worden war, die deutschlandpolitischen Ziele der USA neu zu definieren, Vorschläge für die nächste Außenministerkonferenz zu entwickeln und eine entsprechend revidierte Direktive für OMGUS vorzubereiten ${ }^{26}$.

Das Deutschlandkomitee legte seine Empfehlung erst eine Woche nach der Stuttgarter Rede vor. Seine Zielvorstellungen entsprachen nur noch bedingt dem Stand der internen Diskussion: Die Neutralität eines dauerhaft entwaffneten Deutschland, die wirtschaftliche Gesundung sowohl für Deutschland als auch für Europa und die Entwicklung einer gesunden deutschen Demokratie seien möglichst im Einvernehmen mit der Sowjetunion zu realisieren. Im Falle eines Erfolges dieses letzten Anlaufs zur Verständigung werde dem Kontrollrat bis zum Abschluß eines Friedensvertrages die Aufgabe zukommen, die Entmilitarisierung durchzuführen, die Wirtschaftseinheit und den Wiederaufbau zu koordinieren, entweder durch Zentralverwaltungen, falls die Sowjets das wünschten, oder durch einen „reichsweiten Länderrat“ beim Kontrollrat nach dem Vorbild der amerikanischen Zone. Für ein solches Programm, inkl. der Revision des Industrieniveauplans und einer Regelung der Reparationsfrage, schien Molotows Rede vom 10. Juli Anknüpfungspunkte zu bieten; hinter die Bizone als Alternative und Ersatz, das galt auch hier, würde man jedoch nicht mehr zurückgehen ${ }^{27}$. Akzeptierten die Sowjets das Angebot nicht zu ,vernünftigen“ Bedingungen, war der westliche Alleingang unvermeidlich. Diese Bedingungen hatte Byrnes zwar Punkt für Punkt in seiner Stuttgarter Rede vorgetragen, seine Offerte eines deutschlandpolitischen Neuansatzes richtete sich indes, wie bereits seine Erwiderung auf Molotows Statement in Paris, trotz aller gesamtdeutschen Formeln nicht mehr als ein letztes Angebot an die Sowjetunion. Der Bruch wurde angedroht, aber noch nicht endgültig vollzogen: „Wenn eine völlige [wirtschaftliche] Vereinigung nicht erreicht werden kann, werden wir alles tun, was in unseren Kräften steht, um eine größtmögliche Vereinigung zu sichern. ${ }^{\text {28 }}$ Es war jedoch eine präjudizierende Vorentscheidung getroffen, nämlich die Sowjetunion herauszufordern und die Westlösung nicht länger als ungeliebte Notlösung, sondern als vorteilhaftere Alternative zu betrachten.

Auf diesen Kurswechsel der USA hatten die Briten schon lange gewartet. Sie waren

24 CP, S. 212 ff., 236 ff. TL, Oral History Collection, Riddleberger (1972), S. $80 \mathrm{ff}$.

25 NA, RG 59, 740.00119 Control(Germany)/7-1146 (O’Sullivan an Hilldring) und /7-1646 (Cramer, Claxton und O'Sullivan an Hilldring).

26 NA, RG 59/CED, box 2, folder: G 710 (31. 7. 1946). Mitglieder waren J.W. Riddleberger, J.K. Galbraith, H.P. Leverich und E. Mason. RG 59, 740.00119 Control(Germany)/8-846 und /8-2446. Die Aufgabenstellung: ebenda, /8-746 (Acheson an Murphy); RG 165/014(Germany)/8-1246. Clays Memorandum vom 19. 7. war Ausgangspunkt der Beratungen; im August diskutierte das Komitee mit Clay und Murphy in Berlin. Krieger, Clay, S. 157 ff. Backer, Clay, S. 155 ff. Der Bericht in: RG 59, 740.00119 Control(Germany)/9-1546.

27 Zur Diskussion innerhalb OMGUS vgl. Kindleberger, Letters, S. 57, 59, 64 f.

28 Zitiert nach: Steininger, Deutsche Geschichte, Bd. 1, S. 215. Den Franzosen war von Beamten des State Department mitgeteilt worden, daß die Rede sich nicht gegen die französische Politik richte. AMAE, Y 287, Bl. 215 f. (6. 9. 1946). 
gegenüber der Sowjetunion sehr viel früher auf Distanz gegangen: Seit dem Oktober 1945 wurde intern die „Revision von Potsdam“ diskutiert ${ }^{29}$. Je weniger die Entwicklung in der SBZ eine gemeinsame Politik realisierbar oder wünschenswert erscheinen ließ, desto zurückhaltender waren die Briten gegenüber dem Festhalten der USA an einem prinzipiell gesamtdeutschen Ansatz geworden. Zwar vermutete die britische Botschaft in Moskau, die Sowjetunion habe sich hinsichtlich ihrer deutschlandpolitischen Ziele noch nicht entschieden, sondern wolle sich die Optionen offenhalten; jedoch verlaufe die Entwicklung in Deutschland faktisch zum Nachteil der Westmächte. Die britische Besatzungsverwaltung solle sich daher allen Zentralisierungsansätzen hinhaltend widersetzen, so daß der Kontrollrat bestehen, die deutsche Einheit der Form nach erhalten bleiben und die Verantwortung für mangelnde Fortschritte der Sowjetunion zugeschoben werden könne: „Solch eine Politik würde allerdings darauf hinauslaufen, faktisch, wenngleich nicht unbedingt formal einen wichtigen Teil der Potsdamer Entscheidungen zurückzunehmen. “" ${ }^{30}$ Bei derartigen Überlegungen zur Neubestimmung ihrer Deutschlandpolitik sah sich die Londoner Regierungsbürokratie von den USA eher behindert als gefördert, da Bevin mit Rücksicht auf diese am Potsdamer Abkommen festhielt, solange er nicht sicher sein konnte, ob der ByrnesPlan mehr als nur „hohler Schein“ war ${ }^{31}$. So „wünschenswert“ eine „Überarbeitung“ des Potsdamer Abkommens galt, so wenig stand eine solche angesichts des amerikanischen Zögerns zu erwarten, bestenfalls eine „vernünftige Interpretation“. Obwohl man wußte, daß ein solcher Schritt voraussichtlich den Bruch mit der Sowjetunion provozieren werde, waren die britischen Dienststellen in Deutschland wie in London von der Unvermeidbarkeit baldiger Entscheidungen überzeugt ${ }^{32}$. Zwei Voraussetzungen waren indes zunächst nicht gegeben: der Anlaß, der Bevin zum Umdenken bewog, und die Bereitschaft der USA zu einem Kurswechsel.

Am 3. April diskutierten Bevin, Hynd und Spitzenbeamte aus COGA und Foreign Office die Alternative Einheit oder Teilung Deutschlands. Es zeigte sich, daß auch in London die Ministerialbürokratie und die Planungsstäbe sehr viel früher und radikaler als der Außenminister dazu bereit waren, den Bruch mit der Sowjetunion herbeizuführen und die Kooperation in Deutschland zu beenden. Die große Mehrheit der Teilnehmer sah die Westmächte in der Defensive, propagierte daher die Konsolidierung durch Teilung und warnte vor einer Verzögerung der Entscheidung, sonst werde man eines Tages mit einer deutschen Regierung unter kommunistischem Einfluß konfrontiert sein. Wieder war es allein Bevin, der die pessimistische Einschätzung der Lage zwar weitgehend teilte, der aber noch nicht bereit war, die ihm vorgeschlagenen Lösungswege zu gehen, jedenfalls nicht ohne die USA. Ergebnis der Besprechung war, in einem weiteren Planungspapier die Alternative zu untersuchen: Bildung deutscher Zentralverwaltungen oder Aufbau zonaler Verwaltungen, „einschließlich der Möglichkeit, die drei westlichen Zonen Deutschlands zusammenzuschließen“"33. In der internen Debatte der nächsten Wochen wurde verstärkt die Gefahr einer schleichenden Sowjetisierung Deutschlands und eines deutsch-russischen Zusammengehens be-

29 AMAE, Y 282, Bl. 256 (30. 10. 1945). PRO, FO 800/466/Ger/46/6 und 7 (20. 2. 1946); FO 942/475 (Robertson an Steel, 21.2. 46).

30 PRO, FO 371/55579/C3648 (29. 3. 1946; „cabinet distribution“).

31 PRO, FO 800/466/Ger/46/9 (17. 3. 1946).

32 PRO, FO 371/55586/C1193, C3567, C3216; 55842/C2860, C4360, C5466.

33 Die Ruhrfrage 1945/46, S. 615 ff. 
schworen. Als die beste Möglichkeit zur Sicherung der Ruhr und der Westzonen rückte die Teilung immer stärker in den Vordergrund. Doch noch beschränkte sich Bevin darauf, dem Kabinett Alternativen ohne Lösungsempfehlung vorzutragen, bezog indes, wie Attlee und seine Ministerkollegen, eindeutig gegen die Teilung Stellung, und zwar allein mit dem Argument, die Deutschen würden eine solche niemals akzeptieren und derart lediglich den Russen in die Arme getrieben. Im Grunde hoffte der Außenminister, daß die Sowjetunion durch ihre Politik den Amerikanern die Augen für die Notwendigkeit einer separaten „westlichen Politik“ selbst öffnen werde ${ }^{34}$.

Die britische Strategie war insofern der amerikanischen genau entgegengesetzt. Da Bevin die Fortsetzung „des gegenwärtigen Systems der Verwaltung und Regierung nach Zonen" befürwortete, solange die britische Meinungsbildung und Entscheidungsfindung nicht abgeschlossen war $^{35}$, wurde Frankreich zur Fortsetzung seines Widerstands gegen gesamtdeutsche Lösungen im Kontrollrat ermutigt. Mit der Ruhrfrage hatten die Briten ein noch wirksameres Mittel zur Blockade in der Hand. Die Briten wollten die Ruhrfrage als Hebel einsetzen, um Bewegung in die festgefahrenen Fronten zu bringen, die Revision des Industrieniveauplans zu erzwingen und eine Öffnung der SBZ zu erreichen: Wenn die Sowjetunion als Gegenleistung für die VierMächte-Kontrolle des Ruhrgebiets eine entsprechende Regelung für die sächsische Industrie verweigerte, sei die Legitimation für ein einseitiges Vorgehen gegeben. Mit der Aufkündigung des Potsdamer Abkommens wäre aber auch die „Fassade“ des Kontrollrats nicht länger aufrechtzuerhalten gewesen. Nachdem dieser bzw. die VierMächte-Kooperation insgesamt aufgrund der Konflikte über den Industrieniveauplan, die Reparationen oder den Export-Import-Plan, die die künftige Rolle und Kontrolle des Ruhrgebiets nachhaltig tangierten, ohnehin „in den letzten Zügen“ lägen, dürfte, so übermittelte Strang seine Einschätzung aus Berlin, ohne erhebliche britische Zugeständnisse in dieser Frage auf der Pariser Konferenz „der Bruch ziemlich nahe bevorstehen “36. Diese dramatische Verhärtung in der Haltung der Briten war so offenkundig geworden, daß aufgrund entsprechender Äußerungen von Albu, eines Beauftragten des Deutschlandministers Hynd, bei amerikanischen Stellen der Eindruck entstand, die Politik der Briten scheine darauf hinauszulaufen, „den Kontrollrat platzen zu lassen und sich auf ihre Zone und ihren Sektor von Berlin zurückzuziehen" ${ }^{\text {"37. }}$. Zu einem solch radikalen Schritt war man aber angesichts fehlender deutschlandpolitischer Gestaltungsvorstellungen in London noch nicht in der Lage. Nur durch Vertagung der Entscheidung ließ sich Zeit gewinnen, um eine endgültige Entscheidung über das Ruhrgebiet zu finden; erst die Gründung des Landes Nordrhein-Westfalen bot den Ausweg, den sofortigen Bruch zu vermeiden ${ }^{38}$. Aber solange die interne Meinungsbildung und Entscheidungsfindung nicht abgeschlossen war, konnte der britischen Delegation in Paris nicht daran gelegen sein, trotz der positiven Würdigung des ByrnesPlans durch eine aktive und ausgleichende Verhandlungsstrategie auf einen Erfolg der

${ }_{34}$ PRO, FO 371/55587/C5223 (8. 5. 1946), C5224 (C.M.(46) 43rd Conclusion, 7. 5. 1946; PMM(46)13, 2. 5. 1946); FO 938/348 (Wilberforce, 6. 5. 1946); FO 800/466/Ger/46/16 (Attlee an Bevin, 8. 5 . 1946). Die Ruhrfrage 1945/46, S. 713 ff. (3. 5. 1946), 747 ff. (7. 5. 1946). Zur internen Diskussion vgl. Deighton, Impossible Peace, S. 54-62.

${ }^{35}$ Die Ruhrfrage 1945/46, S. 557 (11.3. 1946).

${ }^{36}$ PRO, FO 371/55424 (CORC, 26. 4. 1946); FO 943/325 (PUSC, 20. 5. 1946).

37 BA, Z 45 F/OMGUS/POLAD/745/27 (Wiesner an Murphy, 23. 5. 1946). Vgl. oben S. 138.

${ }_{38}$ PRO, FO $371 / 55843$ (Orme Sargent, 24. 5. 1946). Die Ruhrfrage 1945/46, S. 186 ff. Vgl. unten S. $415 \mathrm{ff}$. 
Konferenz hinzuarbeiten. Hinzu kam, daß nur ein Scheitern der Konferenz Byrnes die Kooperationsunfähigkeit der Sowjetunion vor Augen führen und zum Einschwenken auf die härtere britische Linie veranlassen würde.

Nach dem erwartungsgemäß enttäuschenden Verlauf der ersten Verhandlungsrunde nutzten die Briten die Konferenzpause, um in London ihre Deutschlanddebatte fortzusetzen $^{39}$. Die Planungen liefen immer stärker auf eine Dezentralisierung bzw. Föderalisierung Deutschlands hinaus ${ }^{40}$, die der Einheit kaum mehr als formal Rechnung trug, sondern durch Verlagerung des Schwergewichts auf die regionalen Gewalten den Trend zur Spaltung forcierte. Bezeichnenderweise galten die hohen Besatzungskosten, die anfangs ein wesentliches Element der britischen Revisionsforderungen gewesen waren, jetzt nicht mehr nur als Belastung, sondern als „Sicherheitsinvestition“: gegen ein Wiedererstarken des Nazismus, gegen die Ausbreitung des Kommunismus und gegen den Rückzug der USA aus Europa ${ }^{41}$. Die Briten verkannten nicht die Risiken einer deutschlandpolitischen Gratwanderung, wenn sie einerseits die Teilung („partition") in Kauf nahmen, um Zeit zur Konsolidierung der eigenen Zone zu gewinnen, wenn sie aber andererseits derart die Spaltung („division“) selbst provozierten, die sie möglicherweise den Rückhalt bei den Deutschen kostete. Daher zielte Bevins Strategie nicht darauf ab, den „vollständigen Bruch mit Rußland“ selbst herbeizuführen oder zu „beschleunigen“; vor einer solch „wichtigen Entscheidung mit weitreichenden Konsequenzen“ schreckte er weiterhin zurück. „Wenn das Potsdamer Abkommen aufgehoben werden müsse, würde er es vorziehen, wenn Rußland dafür die Initiative ergriffe. [...] Wenn ein solcher Bruch eintreten sollte, muß die Verantwortung dafür eindeutig den Russen und nicht uns zur Last gelegt werden." Molotow sollte auf dem $z$ weiten Konferenzabschnitt in Paris ultimativ in einem „show down“ gezwungen werden, seine Karten aufzudecken und klare Auskünfte über die sowjetischen Vorstellungen zur Behandlung Deutschlands als wirtschaftliche Einheit zu geben. Fiel die Antwort unbefriedigend aus, „müssen wir sofort in Deutschland mit dem Ziel handeln, um den Russen eindringlich klar zu machen, wie ernst wir ihr Handeln nehmen, und um den Amerikanern zu zeigen, daß wir ihren Standpunkt unterstützen“. Hier war vor allem daran gedacht, die Sowjets nach amerikanischem Vorbild durch Einstellung der Reparationslieferungen unter Druck zu setzen oder unter Hinweis auf die fehlende Wirtschaftseinheit den Industrieniveauplan aufzukündigen. Diskutiert wurde ebenfalls, wenngleich eher vage, eine engere Kooperation mit der amerikanischen Zone (ebenso mit der französischen, wenn diese sich wider Erwarten anschließen sollte); die Vorstellung einer Bizone wurde immer konkreter. Am 6. Juni stellte Bevin im Kabinett unmißverständlich klar: „Wir könnten zu einer erheblichen Änderung unserer Deutschlandpolitik gezwungen werden." Im Grunde hatte er sich entschieden; doch wieder zögerte er und wollte den Ausgang der Pariser Konferenz abwarten. Er hoffte auf ein Einsehen der Sowjets, doch solange die „Molotow-Mentalität“ in dieser „Diktatur übelster Sorte" vorherrschte, schien wenig Hoffnung für einen Kompromiß zu bestehen $^{42}$.

39 Vgl. die Memoranden und Berichte in: PRO, FO 943/325.

40 PRO, FO $371 / 55587$.

41 PRO, FO $371 / 55588$ (Brief for Prime Minister, Policy for Germany, 21. 4. 1946).

42 PRO, FO 943/325 (Cabinet, 6. 6. 1946; Bevin, 17. 6. 1946); FO 800/501/SU/46/30 (10. 6. 1946). Die Planung in: FO 371/55588/C7267. Die Ruhrfrage 1945/46, S. 876 ff. Vgl. FRUS, 1946/II, S. 454 
Am 10. Juli trug Bevin in Paris sein Ultimatum vor. Zur drohenden Spaltung Europas sei die Vier-Mächte-Kontrolle Deutschlands die einzige Alternative, auch wenn er dieser angesichts der sowjetischen Reparationspolitik nur wenig Chancen einräumte. Unmißverständlich kündigte er an, seine Regierung fühle sich nur unter der Voraussetzung weiterhin an das Potsdamer Abkommen gebunden, daß dieses in seiner Gänze realisiert, die wirtschaftliche Einheit im Hinblick auf den Import-Export-Plan wie auf die Reparationen hergestellt werde. Wenn eine Zusammenarbeit auf der Basis der uneingeschränkten Gegenseitigkeit mit den anderen Zonen nicht zustande komme, müsse England in seiner Zone unilateral vorgehen. Die Wirtschaftseinheit, das war zwei Tage später sein Angebot an Frankreich, sei ihm wichtiger als Zentralverwaltungen ${ }^{43}$. Dieses Ultimatum, das das Angebot einer Bizonenlösung eher zwischen den Zeilen enthielt, wurde von Molotows nachfolgenden Ausführungen zur provisorischen Regierung in Deutschland und zum Friedensvertrag mit Deutschland überdeckt und gewann erst am nächsten Tag an Bedeutung, als Byrnes sich Bevins Analyse anschloß und offiziell sein eigenes Bizonenangebot unterbreitete.

Insgesamt war Bevin mit dem Konferenzergebnis zufrieden. „Nachdem wir lange um den heißen Brei herumgeschlichen sind und zum Fenster herausgeredet haben, sind wir endlich an dem unvermeidlichen Wendepunkt angelangt." Langfristig werde es sich als richtig erweisen, diese Zuspitzung herbeigeführt zu haben. Er und seine Mitarbeiter waren davon überzeugt, daß die Sowjetunion sich auf eine lange Besatzungszeit in Deutschland eingerichtet habe und, nachdem die Chance einer kommunistischen Machtergreifung in Frankreich nicht mehr gegeben sei, auf eine kommunistische Regierung in Deutschland zusteuere. Daher war ein klarer Bruch einem neuen Formelkompromiß vorzuziehen. Doch der Außenminister schreckte abermals vor den Konsequenzen seiner Überzeugungen wie seiner bisherigen Politik zurück. Er warnte am 15. Juli das Kabinett, „es wäre ein Fehler zu diesem Zeitpunkt, wenn wir uns unwiderruflich auf eine Maßnahme festlegten, die eine vollständige Spaltung zwischen Ost- und Westdeutschland implizierte“, indem nämlich England das amerikanische Bizonenangebot annehme, ohne - das war der Kern seiner Unsicherheit - die Gewißheit zu haben, daß die USA diese Politik langfristig mittrugen ${ }^{44}$. Um die Bedeutung ihres Kurswechsels zu kaschieren und eine formale Aufkündigung des Kontrollrats zu vermeiden, griffen die Briten verstärkt auf die amerikanische Interpretation des Kontrollabkommens zurück, daß angesichts des Fehlens einer Vier-Mächte-Einigung die unilaterale Handlungsfreiheit in der eigenen Zone und das Recht zu bilateralen Arrangements gegeben sei. Gleichwohl bedurfte es am 23. Juli auf einer Besprechung von Bevin, Hynd, Robertson und verschiedenen Spitzenbeamten erheblicher Überzeugungsarbeit, bis ersterer seine Vorbehalte gegenüber der Bizone endlich aufgab. Da er weiter zweifelte, ob ein „angelsächsischer Block“ dazu beitragen werde, „uns in die

(Bevin an Byrnes, 29. 5. 1946). Zu den Vorbereitungen der Briten gehörte, wie bei den USA, eine Liste mit sowjetischen Verstößen gegen das Potsdamer Abkommen. PRO, FO 371/55589/C8642 (22. 7. 1946). Es gab nur wenige warnende Stimmen: „I am not sure whether it is really the right policy to provoke a very early show-down with the Russians about economic unity." Die Sowjetunion werde sich weigern, und „we shall have crossed the Rubicon“. FO 943/325 (Turner, 15.6. 1946). Nach französischen Berichten war Bevin bereits jetzt entschlossen, im Notfall eine Bi- oder Trizone zu gründen. AMAE, Y 147, Bl. 22 (13.6. 1946).

43 FRUS, 1946/II, S. 864 ff., 896, 911.

44 PRO, FO 371/55843/C7729, 55844/C7885, C8111, C9932; FO 943/325. Die Ruhrfrage 1945/46, S. 937 ff. Vgl. Deighton, Impossible Peace, S. $105 \mathrm{ff}$. 
beste Position für Verhandlungen mit den Russen zu bringen“, wies er das Kabinett am 25. Juli abermals nachdrücklich darauf hin, ein Eingehen auf das amerikanische Angebot werde die vorhandenen Tendenzen zur Spaltung Europas beschleunigen. Aber, so versuchte er sich selbst zu trösten: „Die vorgeschlagene Maßnahme wird die Dinge nicht schlimmer machen, als sie gegenwärtig sind“; sie könnte im Gegenteil einen „heilsamen Effekt“ auf die Sowjets ausüben ${ }^{45}$. Doch im Grunde täuschte sich niemand darüber hinweg, daß das Gegenteil der wahrscheinlichere Fall war. Das amerikanische Angebot komme einem „offenen Eingeständnis“ gleich, daß die VierMächte-Verwaltung gescheitert sei. „Wenn Deutschland ökonomisch geteilt werden solite, wird die politische Teilung beinahe sicher folgen, auch wenn das nicht notwendigerweise sofort der Fall zu sein braucht." Da voraussichtlich die Sowjetunion alles tun werde, um einen Erfolg der Bizone zu verhindern, werde diese nicht, sozusagen auf Umwegen, zur wirtschaftlichen Einheit Deutschlands zurückführen, sondern vielmehr die Entfremdung verstärken, die Sowjets nicht kompromißgeneigter, sondern konfliktbereiter machen ${ }^{46}$.

\section{Frankreichs Niederlage: Die Verhinderung der politischen Einheit, die Ruhrfrage und die Gründung der Bizone}

In Paris war im Zuge der Auseinandersetzungen um die Zentralverwaltungen vereinzelt die Erkenntnis gewachsen, daß die Forderung nach Abtrennung von Saar, Ruhr und Rheinland nicht durchzusetzen war $^{47}$. Im Februar hatte die Regierung unter amerikanischem Druck die Gleichrangigkeit von Saar- und Ruhrfrage aufgegeben und im Interesse der Wirtschaftseinheit, für die man „stets“ eingetreten sei, Anfang Mai akzeptiert, „daß Deutschland in seinen gegenwärtigen Grenzen provisorisch als eine wirtschaftliche Einheit behandelt werde “48. Während die französische Außenpolitik einerseits die Kooperation mit der Sowjetunion suchte, um ein zentralistisch organisiertes Deutschland zu verhindern, war sie andererseits auf die Unterstützung der westlichen Alliierten angewiesen, wollte sie ihre Teilhabe an der Kontrolle des Ruhrgebiets sichern. Eine anglo-amerikanische Koalition, die einen derartigen Zugriff behinderte und die sowjetische Hilfestellung entwertete, konnte keinesfalls in ihrem Interesse liegen. Frankreichs Dilemma im Sommer 1946 lag darin, daß man zu lange auf die wechselseitige Blockade von Anglo-Amerikanern und Sowjets gesetzt hatte und nun nicht effektiv zu reagieren vermochte, als durch die amerikanische Initiative auf der Pariser Konferenz Bewegung in die deutsche Frage kam.

Auch in Paris war es der Außenminister, der nicht bereit war, die vorsichtige Kurskorrektur der Pragmatiker nachzuvollziehen. Bidault wiederholte in seinem Konferenzpapier vom 25. April 1946 seine Maximalpositionen hinsichtlich der Saar, der Ruhr und des Rheinlands, obwohl nach den Vorverhandlungen mit den Westmächten

45 PRO, FO 371/55589/C8643 (23. 7. 1945); FO 943/325 (73rd Cabinet, 25. 7. 1946). Vgl. AMAE, Y 288, Bl. 357 (25. 10. 1946).

46 PRO, FO $371 / 55700$ (34. CONL, 23. 7. 1946); FO 800/466/Ger/46/26 (25. 7. 1946); FO 1046/94 (Troutbeck, 1.8. 1946).

47 AMAE, Y 286, Bl. 279 (Laffon, 8. 2. 1946). Vgl. Kettenacker, Krieg, S. 434 ff.

48 AO, Berlin/3270/1/2132 (2. 5. 1946; meine Hervorhebung). Vgl. AMAE, Y 377, Bl. 9 (15. 6. 1946), 11 (20.6. 1946). 
eine Annäherung der Standpunkte nicht zu erwarten war. Während Briten und Amerikaner die Wirtschaftseinheit nur im Zusammenhang mit der territorialen Einheit Deutschlands verhandeln wollten, sahen Franzosen und Sowjets die Ruhrfrage als qualitativ anders an. Sie forderten, dieses Spezialproblem im Sinne ihrer nationalen Interessen vor der Behandlung des Schicksals (Rest-)Deutschlands zu klären. Der anglo-amerikanische Versuch, an die Reparations- und Rekonstruktionsinteressen der beiden zu appellieren, stieß ins Leere: Bidault hob den rein politisch definierten Primat der Sicherheit hervor, hinter dem die Frage der wirtschaftlichen Rationalität zurückstehen müsse. Die Franzosen erkannten die Tragweite des Byrnes-Plans, ihr Hauptaugenmerk blieb aber auf die „unbegrenzte“ Besetzung eines militärisch und wirtschaftlich entmilitarisierten Rumpf-Deutschlands gerichtet. Da die USA unter Erfolgszwang zu stehen schienen, wenn Byrnes die deutschlandpolitische Initiative von den Sowjets zurückgewinnen wollte, glaubte Bidault, auf seiner Maximalforderung nach Abtrennung von Rheinland, Ruhr- und Saargebiet beharren zu können ${ }^{49}$.

Während der Außenminister starr an seiner Linie festhielt, eröffnete Chauvel, der schärfste Kritiker der Außenpolitik Bidaults im Quai d'Orsay, den Anglo-Amerikanern inoffiziell seine „persönliche Meinung“. Er ermunterte die USA zur Bizonengründung, da es für Frankreich trotz eines offiziellen Dementis gar keine Alternative gebe, als „aus sehr praktischen Gründen“ mit der Bizone zu kooperieren. Das zu erwartende Scheitern der Außenministerkonferenz werde die ökonomische und politische Spaltung Deutschlands nach sich ziehen. Falls dann die britische und amerikanische Zone „eng zusammenarbeiteten“, so mußte er einräumen, werde sich die französische Regierung aus innenpolitischen Rücksichten einem derartigen Arrangement aber nicht anschließen können. Er drängte daher auf ein zurückhaltenderes Vorgehen: Wenn die künftige Bizone die Bande mit der französischen Zone durch pragmatische Einzelfallregelungen verfestige, könnten diese später unter einer anderen Regierungskonstellation leichter formalisiert werden ${ }^{50}$. Ebenso übersah auch Saint-Hardouin die Zwänge nicht, sich mit der Bizone zum eigenen Vorteil zu arrangieren $^{51}$. Die GFCC warnte ihrerseits im Juni 1946 die Regierung in Paris wiederholt, die Angelsachsen bereiteten eine Art Befreiungsschlag bzw. ein Einschüchterungsmanöver vor, da sie sich nicht länger von den Sowjets „düpieren“ lassen wollten. Angesichts der zunehmenden Selbstisolierung der SBZ rücke die Möglichkeit einer Revision des Potsdamer Abkommens immer näher; dann werde als automatische Folge die Fusion der amerikanischen und britischen Zone kaum mehr zu verhindern sein ${ }^{52}$. Da Frankreich im Falle einer politischen und/oder wirtschaftlichen Teilung Deutschlands nur verlieren konnte, drängte auch Koenig auf „Modifikationen“, um die Deutschlandberatungen auf der Konferenz nicht vollständig scheitern zu lassen und seine Vetoposition im Kontrollrat nicht zu verlieren ${ }^{53}$. Es mehrten sich die internen War-

49 AMAE, Y 356, Bl. 290 ff., 346 ff., 404 ff., 437 ff. FRUS, 1946/II, S. 109 ff., 394 ff.

so NA, RG 59, 740.00119 Control(Germany)/6-1146 und /6-2246. Chauvel wiederholte die Ermunterung gegenüber den Briten, nachdem die Entscheidung gefallen war. PRO, FO 371/55589/C8423 (23.7. 1946, Cabinet distribution). Ebenso im September erneut gegenüber den Amerikanern. FRUS, 1946/V, S. 566 f., 605.

si AMAE, Y 289, Bl. $130 \mathrm{ff}$.

s2 AMAE, Y 286, Bl. 24 ff., 43 ff., 87 f., 147; Y 460 (Synthèse mensuelle, Mai 1946, S. 6).

53 AMAE, Y 286, Bl. 147 f. (3. 7. 1946); Y 370, Bl. 145 (10.6. 1946). 
nungen, das Land könne durch eine Verhinderungspolitik seine Ziele nicht verwirklichen, sondern werde nur die Bündnispartner verärgern; nicht Spaltung, sondern Kooperation diene den nationalen Interessen ${ }^{54}$. Eine „neue Überprüfung“ der gesamten Deutschlandpolitik schien um so dringender, als die Sowjets die Forderung nach gemeinsamer Verwaltung der Ruhrkohle und nach Wiederaufnahme der Reparationslieferungen bereitwillig unterstützten, aber jede Abtrennung der Ruhr kompromißlos ablehnten ${ }^{55}$. Die Isolation war vollständig, als Molotow mit seinem Statement vom 10. Juli die sowjetisch-französische „Aktionsgemeinschaft“ aufkündigte und damit das Argument der Regierung in Paris, innenpolitisch auf die KPF Rücksicht nehmen zu müssen, entwertete, nachdem die französischen Kommunisten - zur Verwirrung der Anglo-Amerikaner - bislang für, die deutschen Kommunisten gegen die Abtrennung des Ruhrgebiets votiert hatten ${ }^{56}$.

Frankreich sah sich gezwungen, zur Wahrung seiner Interessen die Deutschlandfrage weiter von der Ebene einer prinzipiellen Gesamtregelung der politischen Einheit auf die Ebene pragmatischer ad hoc-Lösungen zu verlagern, um auf dem Wege zumindest die wirtschaftliche Einheit zu erhalten: Diese „hängt nicht von der Schaffung einer deutschen Zentralverwaltung ab, sondern von einem wirtschaftlichen Abkommen zwischen den vier Alliierten“; die von Deutschland während des Krieges zerstörten Länder hätten ein Recht darauf, nicht durch die Uneinigkeit der Alliierten bestraft zu werden ${ }^{57}$. Bidault wiederholte auf der Pariser Konferenz am 12. Juli abermals die alten Forderungen, deren Erfüllung - außer im Falle der Saar - aber nicht mehr Vorbedingung weiterer Vereinbarungen war, sondern mehr auf die Befriedigung der „akuten Bedürfnisse“ bezogen wurde. Er akzeptierte daher jetzt im Interesse der „vorläufigen“ Herstellung der Wirtschaftseinheit zum einen Bureaux alliés mit deutschem Personal beim Kontrollrat, solange deren Zuständigkeitsbereich nicht auf die Saar ausgedehnt werde. Zum zweiten forderte er die Steigerung der Kohleproduktion, nicht zugunsten des deutschen Kohleverbrauchs oder des Exports, sondern zugunsten der Lieferungen an Frankreich. Zum dritten empfahl er eine Ausweitung des Interzonenhandels, um durch eine bessere innerdeutsche Ressourcenverteilung die Belastung der Besatzungsmächte zu reduzieren und den Export von Kohle zu erleichtern ${ }^{58}$. Der Vorstoß blieb nicht ohne Erfolg: Briten und Amerikaner akzeptierten die faktische Abtrennung der Saar ohne französische Gegenleistungen. Der Kontrollrat setzte auf Anweisung der Außenminister ein „Komitee von Kohleexperten“ ein und regelte die Ausweitung und Erleichterung des Interzonenhandels.

Doch die Hoffnung trog, mit einer solchen Politik der „guten Nachbarschaft" ${ }^{\text {"59 }}$ die drohende politische und wirtschaftliche Isolierung der eigenen Zone durch die Bizone verhindern oder zumindest abmildern zu können. Wenn die Franzosen bemängelten, die Gründung des Landes Nordrhein-Westfalen und der Bizone beeinträchtige die

54 AMAE, Y 286, Bl. 156 f. (Saint-Hardouin, 5. 7.), 285, 290 (Laffon, 26. 7. 1946), 333 ff. (Couve de Murville, 31. 7. 1946).

ss AMAE, Y 377, Bl. 11 (20.6. 1946).

s6 NA, RG 59, 740.00119 Control(Germany)/7-1146 (Caffery an Byrnes). Vgl. FRUS, 1946/V, $465 \mathrm{f}$. (12.7. 1946).

57 AO, Berlin/3270/1/2132 (29.6. 1946).

58 FRUS, 1946/II, S. 909 f. (12. 7. 1946).

s9 AMAE, Y 377, Bl. 73 (30. 7. 1946). 
Kompetenzen des Kontrollrats ${ }^{60}$, so ließ das erkennen, wie sehr sie von der Bizone eine Untergrabung ihrer gesamtdeutschen Vetoposition befürchteten. Die Regierung in Paris wußte, daß ihre Blockadepolitik der ersten Monate vollständig gescheitert und die Zeit gekommen war, zur Wahrung der eigenen besatzungspolitischen und Reparationsinteressen im Kontrollrat mehr Flexibilität und Entgegenkommen zu zeigen ${ }^{61}$. Vorgeblich war auch Bidault inzwischen zu der Einsicht gelangt, daß die starre Haltung in der Ruhrfrage ein von de Gaulle ererbter „Fehler“ sei: „Während er seine eigenen Ansichten zum deutschen Problem modifiziert habe und es offenkundig im Interesse Frankreichs liege, eine Verständigung gerade mit den Amerikanern und Briten zu erzielen, sei es für ihn unmöglich, vor den nächsten Wahlen einen Kurswechsel in seiner Politik zu vollziehen. Nach den Wahlen jedoch, [...], werde er auf eine Modifizierung der französischen Politik hinarbeiten, die für Amerika und Großbritannien akzeptabel sei." ${ }^{\text {62 }}$ Das lief auf eine neuerliche Vertagung der Grundsatzentscheidungen hinaus. Derartige Vertröstungen waren auch nicht neu. Aber das ständige Schwanken Bidaults, die Anerkennung der strukturellen Abhängigkeit von den Anglo-Amerikanern schwächten die französische Verhandlungsposition und setzten den Minister verstärkten Pressionsversuchen aus. Vor allem die Briten waren entschlossen, trotz allen Verständnisses für die Zurückhaltung der französischen Politik ihre Trumpfkarte, die Kontrolle der Ruhr, voll auszuspielen ${ }^{63}$.

Wie schwach die französische Position war, ließ sich an der Verbitterung erkennen, mit der in Berlin bei den Militärs wie bei Saint-Hardouin der neue Kurs der USA und Englands aufgenommen wurde. Koenig bedauerte, daß ausgerechnet am Jahrestag von Potsdam die westlichen Alliierten davon sprächen, „Deutschland politisch wiederherzustellen“, und verlangte von seiner Regierung, Garantien gegen die Risiken dieser voreiligen Beschlüsse zu fordern. Saint-Hardouin interpretierte die Bizonenstrategie als den Versuch, den Sowjets die Schuld für die Spaltung Deutschlands zuzuschieben. Die USA würden sich notfalls auch auf Kosten Frankreichs mit der Sowjetunion auf einen Anschluß der SBZ an die Bizone einigen, da „bei ihnen die Animosität gegenüber den Russen eine Rachsucht gegenüber uns nicht ausschließt". Ausgerechnet er beklagte nun den „Partikularismus der Zonen“, die „sehr große Freiheit" der Kommandeure in ihren Zonen, die „Schwäche der zentralen alliierten Behörden“64. Doch der betonte Versuch, im Kontrollrat Übereinstimmung mit den Sowjets gegen die Bizone und für die Ausweitung des Interzonenhandels zu demonstrieren, trug nicht weit. Denn die Sowjetunion wollte, taktisch wenig geschickt, weder Frankreichs Ansprüche auf die Saar akzeptieren, noch deren Vorschlag zur Errichtung von Bureaux alliés unterstützen. Solange Franzosen und Sowjets die politische Einheit als Beeinträchtigung ihrer deutschlandpolitischen Autonomie betrachteten und die wirtschaftliche Einheit nur zu ihren sehr spezifischen Bedingungen akzeptieren wollten, solange die Franzosen nur eine alliierte, aber keine deutsche Einheit befürworteten, war mehr

60 AMAE, Y 377, Bl. 86 ff. (SGAAA, 31. 7. 1946). Diese Auffassung vertraten nachdrücklich auch die Sowjets. Belezki, Politik, S. 54, 110.

61 PRO, FO 943/325 (Chauvel, 9. 8. 1946). AMAE, Y 282, Bl. 391 ff. (Massigli, 30. 10. 1946).

62 FRUS, 1946/V, S. 596 (30. 8. 1946). AMAE, Y 289, Bl. 7 (Bidault, 1. 11. 1946). Auch: NA, RG 200/ Clay, box 2, folder: French Relations (21.8. 1946).

${ }^{63}$ FRUS, 1946/V, S. 603-10. Die Demarche der USA vom 24. 7. in: FRUS, 1946/V, S. 583 ff.; die französische Antwort ebenda, S. 596 ff. Zur britischen Anfrage vom 31. 7.1946 vgl. PRO, FO 943/325.

64 AMAE, Y 287, Bl. 103 (10. 8.), 161 (30. 8. 1946); Y 288, Bl. $391 \mathrm{ff}$. 
als eine brüchige Verhinderungskoalition nicht denkbar, die der Dynamik der Bizonengründung nichts entgegenzusetzen vermochte. Die letzten Illusionen verflogen bald: Nachdem sich die Hoffnung zerschlagen hatte, die Briten würden die Bizone doch noch ablehnen ${ }^{65}$, war die französische Verzögerungstaktik endgültig gescheitert. Saint-Hardouin, Sergent und de Leusse wurden im November nach New York delegiert, um dort dank ihrer persönlichen Beziehungen die britisch-amerikanischen Bizonenverhandlungen so zu beeinflussen, daß die Entscheidungen nicht „zu endgültig“ ausfielen $^{66}$.

\section{Die Sowjetunion am Scheideweg: Reparationen oder Sicherheit?}

Bemerkenswert an Molotows Statement vom 10. Juli war weniger der Inhalt als seine propagandistische Verwertung. Der vermeintliche Auftakt einer sowjetischen Offensive war die eher hilflose Reaktion auf die westlichen Vorstöße vom Tage zuvor. Die Sowjetunion mußte sich entscheiden, ob sie eine Öffnung nach Westen oder eine Politik der Abgrenzung in Deutschland betreiben wollte. Einerseits hatten die Gründung der SED und die Erweiterung des Blocks durch den FDGB, der Abschluß von Bodenund Industriereform ihre Position in der SBZ konsolidiert. Andererseits beobachtete sie mit wachsendem Mißtrauen, daß die USA und Großbritannien immer energischer auf eine Lösung zusteuerten, die ihr zum Nachteil gereichen mußte ${ }^{67}$. Angesichts „der Verschärfung aller politischen Umstände in Deutschland“, die der Politische Berater Semjonow nach Moskau meldete ${ }^{68}$, waren auch dort die Weichenstellungen längst erfolgt. Während Molotow in Paris an den Einheitswillen der Deutschen appellierte, befahl die SMAD die Errichtung der Sowjet AGs innerhalb von 14 Tagen ${ }^{69}$.

Mit dieser Entscheidung steuerte die Sowjetunion unvermeidlich auf die Teilung Deutschlands zu. Um freie Hand für die Ausbeutung der eigenen Zone zu gewinnen, konnte sie an gesamtdeutschen Lösungen nicht länger interessiert sein, die diesen Zugriff beeinträchtigten. Daher stieß das Angebot des Byrnes-Plans ins Leere. Denn in Verbindung mit dem Reparationsstopp schien dieser auf eine erhebliche Modifikation, wenn nicht Revision des Potsdamer Abkommens hinauszulaufen und ein Abrükken von Industrieniveauplan und industrieller Entwaffnung zu signalisieren. Die beiden sowjetischen Prioritäten, Sicherheit und Reparationen, drohten zunehmend in Widerspruch zu geraten: Sicherheit vor Deutschland und vor einem antisowjetischen „Westblock“, der das Potential der Ruhr einbezog ${ }^{70}$, waren nur durch „Einheit“, durch Kooperation mit den Westmächten zu erreichen, die dafür den (vorübergehenden) Verzicht auf Reparationen verlangten. Der Byrnes-Plan, der dieses Dilemma der Sowjetunion (und Frankreichs!) überwinden sollte, war inakzeptabel, weil er im Hinblick

65 AMAE, Y 287, Bl. 272 (CCFA, 10.9. 1946).

66 AMAE, Y 286, Bl. 333 ff.; Y 288, Bl. 370 ff. (Koenig, 30. 10. 1946), 391 ff. (Massigli, 30. 10. 1946); Y 289, Bl. 43, 66 ff.

${ }^{67}$ Zum sowjetischen Interesse an Gerüchten über die bevorstehende Gründung einer Bizone vgl. AMAE, Y 286, Bl. 87 f. (15.6. 1946).

68 Zitiert nach: Laufer, Ursprünge, S. 167 (Anm. 26).

69 ThHStA, MWA/420, Bl. 64.

70 Ende September 1945 hatte Molotow sich bei Bidault beschwert, Frankreich unterstütze die Sowjetunion nicht gegen diese Entwicklung. AMAE, Y 134, Bl. $117 \mathrm{f}$. 
auf die künftigen Reparationslieferungen dem Wiederaufbau Deutschlands Priorität einräumte und weil er die Sowjetunion zu Wohlverhalten und Kooperation gezwungen hätte, um ihre Ansprüche nicht zu gefährden. Ziel des Byrnes-Plans sei, so analysierte das Außenministerium, nicht nur die „schnellstmögliche Beendigung der Besetzung Deutschlands“, sondern auch die „Schwächung des Einflusses der Sowjetunion in den Deutschland betreffenden Fragen“. Da in der von Byrnes zur Durchführung seines Planes angeregten Kontrollkommission das Mehrheitsprinzip das Veto ersetzen sollte, würde der sowjetische Vertreter „sich immer in der Minderheit befinden“. Empfohlen wurde daher die Ablehnung des Plans mit der Begründung, da die Potsdamer Beschlüsse zur Entmilitarisierung nicht erfüllt seien, komme die Einsetzung einer solchen Kontrollkommission „verfrüht" ${ }^{\text {"71 }}$.

Schon im ersten Vorgespräch zwischen Byrnes und Molotow am 28. April 1946 in Paris hatte letzterer diese Position vorgetragen: Der Byrnes-Plan nehme den zweiten Schritt vor dem ersten in Angriff, wenn er die Entwaffnung Deutschlands zu garantieren verspreche, die bislang noch gar nicht durchgeführt sei. „Der amerikanische Vorschlag sieht nicht die Entwaffnung Deutschlands vor, sondern die Vertagung dieser Entwaffnung." Erst nach der Durchführung der früheren Beschlüsse könne man über den Modus zukünftiger Kontrollen reden. Er wiederholte seinen Antrag vom Dezember 1945, durch den Kontrollrat den Stand der Entwaffnung in den Zonen überprüfen zu lassen und währenddessen den Byrnes-Plan im Außenministerrat zu diskutieren ${ }^{72}$. Der tote Punkt war erreicht, zumal auch Bevin und Bidault keineswegs uneingeschränkt auf die Vorstellungen Byrnes' eingingen. Dieser war enttäuscht und verärgert, aber das konnte ihn nicht davon abhalten, einen erneuten Vorstoß zu unternehmen. Er überraschte am 13. Mai seine Kollegen mit der Mitteilung, Clay habe im Sinne der Forderungen Molotows Anweisung erhalten, im Kontrollrat den Stand der Entwaffnung durch eine Kommission klären zu lassen.

Am 17. Mai debattierte das Koordinationskomitee in Berlin über die Einsetzung einer Inspektionskommission, die den Stand der Entmilitarisierung in allen vier (und nicht nur den westlichen) Zonen überprüfen sollte. Bereits am 23. Mai gab es die üblichen Differenzen, als die Westmächte neben der personellen und materiellen Abrüstung auch die ökonomische einzubeziehen verlangten. Zum einen verdächtigten sie die Sowjetunion, in ihrer Zone die im Potsdamer Abkommen verbotene Produktion von Rüstungsgütern für den eigenen Gebrauch zu betreiben, obwohl sie selbst Verstöße eingestanden ${ }^{73}$; zum anderen wollten sie die Bereitschaft der Sowjets zur Öffnung ihrer Zone für eine solche Kommission testen - und sich einen propagandistischen Vorwand für den Fall verschaffen, daß diese sich einer Kontrolle verweigerten. Erwartungsgemäß lehnte der sowjetische Vertreter eine Kontrolle der industriellen Abrüstung auch in der SBZ mit dem Argument ab, bislang seien in den Westzonen keine derartigen Maßnahmen eingeleitet worden, so daß sich die (Rüstungs-)Industrie in den Westzonen „im wesentlichen in demselben Zustand wie bei Ende des Krieges“

7 Zitiert nach: Laufer, Auf dem Wege, S. 31 f. (Nowikow an Stalin, 29. 5. 1946). Die SMAD und der Politische Berater Semjonow unterstützten diese Analyse.

72 FRUS, 1946/II, S. 147, 167 ff. Vgl. FRUS, 1945/II, S. 702 ff.

73 NA, RG 59, 740.00119 Control(Germany)/6-2646 (Assistant Chief of Staff, G-2, General Sibert, Alleged Soviet Violations of the POTSDAM Protocol and Control Council Directives, zur Produktion von V-Waffen, Flugzeugen und Lenkwaffen in der SBZ). PRO, FO 800/466/Ger/46/52 (7. 11. 1946). Frankreich bestand auf einer Fortführung der Sprengstoffproduktion bis zum 1. 1. 1949. 
befinde. Bezeichnend war der Nachsatz, die Wiederaufnahme der Reparationslieferungen sei das wirksamste Mittel, um auf dem Gebiet Fortschritte zu erzielen; der Kontrollrat solle daher zunächst festlegen, was als Kriegsindustrie anzusehen, was zu zerstören und was zum Zwecke von Reparationen zu demontieren sei $^{74}$. Damit war das Problem auf die Ebene des Taktierens um Propagandavorteile und Zeitgewinn verlagert worden. Als die Sowjets Anfang August einlenkten, war der politische Schaden nicht mehr reparabel.

Die Initiative zur Entwaffnung Deutschlands hatte die Sowjetunion am 30. August $1945 \mathrm{im}$ Kontrollrat ergriffen und - nachdem ihr Mißtrauen durch das Verwirrspiel um die Regierung Dönitz geweckt worden war $^{75}$ - die verzögerte Demobilmachung der Wehrmacht in der britischen Zone zum Anlaß für eine immer heftigere Kritik genommen, die zum ersten offenen Konflikt im Kontrollrat führte. Zwar konnte der sich auf eine Reihe von Maßnahmen zur Entmilitarisierung einigen, doch erregte weniger die Existenz der „Dienstgruppen“, Minenräumdienste oder Kriegsgefangenenlager das Mißtrauen der Sowjets ${ }^{76}$, sondern die Beobachtung, daß deutsche Stäbe mit „Selbstverwaltungs“-Rechten oder „beratenden“ Aufgaben weiter bestanden; wichtig seien die Strukturen, die - so Schukow offen - jederzeit auf Kriegsverhältnisse umgestellt werden könnten ${ }^{77}$. Sokolowski reagierte heftig, als er am 23 . Oktober erfuhr, daß deutsche, wenngleich unbewaffnete Wehrmachtseinheiten in der britischen Zone bestanden, obwohl das Koordinationskomitee seit dem 16. Oktober über deren Auflösung debattierte. Da erläuternde Briefe Montgomerys vom 26. Oktober bzw. 8. November nicht befriedigten, legte Schukow am 20. November die Frage dem Kontrollrat selbst vor. Der amerikanische Vertreter McNarney zeigte sich „überrascht“, daß die Briten auch deutsche Stäbe beibehalten hatten, und kritisierte das offen als Verstoß gegen Potsdam. Als sich Koenig der Kritik anschloß, mußte Montgomery sich verpflichten, eine Aufstellung der Einheiten und Mannschaftsstärken vorzulegen sowie einen Zeitplan für deren endgültige Auflösung ${ }^{78}$. Während Sowjets und Franzosen kaum noch Kriegsgefangene oder gar deutsche Einheiten in ihren Zonen zu haben erklärten, meldeten die USA 2043 „Arbeitsdienst“-Einheiten mit 530.000 Angehörigen. In der britischen Zone gab es noch 656.000 Kriegsgefangene, von denen 225.000 für

74 FRUS, 1946/V, S. 559-65.

75 Schukows Bericht, daß ,the British are keeping the German troops in a state of combat readiness and establishing cooperation with them", war der letzte Anlaß für Stalin, die Verhaftung von Dönitz zu betreiben. Schukow, Erinnerungen, S. 636 ff. Vgl. Potsdamer (Berliner) Konferenz 1945, S. 312 f.

${ }^{76}$ Auf der Moskauer Außenministerkonferenz im März 1947 drängte die Sowjetunion abermals auf die Auflösung der Dienstgruppen in der britischen Zone bis zum Ende des Jahres. Die Minister beauftragten den Kontrollrat, die Entmilitarisierung bis zum 31. 12. 1948 abzuschließen. Borgert, Dienstgruppen, S. 99. BA, Z 45 F/OMGUS, 2/93-1/8. Übersichten in: 2/93-1/7, 8 und 10.

77 Demobilmachungs-Einheiten waren in einer „Bemerkung“ zum Kontrollratsgesetz Nr. 8 vom 30. 11. 1945 ausdrücklich ausgenommen, das die militärische Ausbildung und das Tragen von Militäruniformen untersagte, nicht aber die 20.000 deutschen Soldaten, die in britischem Gewahrsam unter Waffen standen. Smith, Churchill's German Army, S. 90.

$78 \mathrm{BA}, \mathrm{Z} 45$ F/OMGUS, 2/108-3/1; 2/118-1/2-4 (CORC/M(45)11-27); 2/118-1/5-16 (CORC/P (45)52, 92, 114 und 191). Der Schukow-Bericht und Montgomerys Antwort vom 8.11. 1945 sowie weitere Materialien in: 2/93-1/7 und 8. Die britische Übersicht in: DBPO, I, 2, S. 839 ff. Vgl. Montgomery, Memoirs, S. 403-13. Intern gestanden die britischen Militärs die Berechtigung der sowjetischen Intervention ein. DBPO, I, 2, S. 797. DBPO, I, 5, S. 410 f., 415 f. Smith, Churchill's German Army, S. 93 ff. Am 13. 5. 1946 deutete Byrnes dem verärgerten Bevin an, er zweifele daran, daß die Briten die Entmilitarisierung in ihrer Zone im vereinbarten Sinne durchführten, äußerte aber zugleich den Verdacht, die Sowjets wollten mit ihren Attacken nur von eigenen Verstößen ablenken. PRO, FO 800/466/Ger/46/17. 
Reparationsleistungen in England vorgesehen waren. Zwar betonte Schukow diplomatisch, er wolle Montgomery nicht unterstellen, daß er „gegen Rußland Krieg zu führen wünsche ${ }^{479}$, aber diese Äußerung fiel nicht ohne Bedacht. Da sich unter den Kriegsgefangenen in der britischen Zone 126.000 Nicht-Deutsche befanden, da erst aufgrund der sowjetischen Proteste die Rekrutierung von Displaced Persons für eine polnische Division eingestellt wurde, vermutete Sokolowski hinter diesen Aktivitäten die Rekrutierung einer neuen Wlassow-Armee ${ }^{80}$. Am 20. Dezember beschuldigte Schukow während einer „hitzigen“ Debatte im Kontrollrat die Briten, die Aufrechterhaltung von deutschen und (das fügte er ausdrücklich hinzu) polnischen Einheiten unter britischer Oberleitung erfolge kaum ,in Übereinstimmung mit der vorrangigen Aufgabe der Besatzung“, damit weitergehende Absichten unterstellend. Am folgenden Tag akzentuierte Molotow diesen Aspekt auf der Moskauer Außenministerkonferenz noch schärfer, indem er auch die Existenz von ungarischen, lettischen und litauischen Verbänden kritisierte. Aber obwohl er die Angelegenheit wiederholt als „Frage von großer Bedeutung für die Sowjetunion“ bezeichnete ${ }^{81}$, machten die Sowjets den Punkt zu keiner Zeit zum Gegenstand öffentlicher Propaganda-Angriffe gegen die Briten.

Nachdem die Debatten im Kontrollrat keine Fortschritte erbracht hatten, forderte die Sowjetunion am 10. Juli auf der Pariser Außenministerkonferenz abermals die Einsetzung einer Entmilitarisierungskommission, da ihr Antrag vom Dezember 1945 ebenso unbeachtet geblieben sei wie der Vorstoß im Kontrollrat vom Juni 1946, einen gesamtdeutschen Plan zur Beseitigung des deutschen Kriegspotentials zu erarbeiten ${ }^{82}$. Nachdem am 10. April 1946 durch Gesetz Nr. 23 die Errichtung militärischer Bauten in Deutschland verboten worden war, das alle vier Mächte noch am wenigsten beeinträchtigte, konnte sich jetzt der Kontrollrat am 20. August 1946 auf das Gesetz Nr. 34 einigen, in dem die Auflösung der deutschen Wehrmacht noch einmal verkündet wurde, obwohl das bereits in der Proklamation Nr. 2 vom 20. September 1945 „vollständig und endgültig“" angeordnet worden war. Am 2. Oktober 1946 kam es mit Direktive Nr. 39 zu einer Einigung über die Kontrolle der industriellen Entwaffnung durch Vier-Mächte-Inspektionsteams und zur Einführung einer Berichtspflicht über die erhaltenen Rüstungsbetriebe ${ }^{83}$. Am 29. Dezember 1946 erfolgte „zur Verhinderung der Wiederaufrüstung Deutschlands“ das Gesetz Nr. 43, das die Herstellung, die Ein- und Ausfuhr, Beförderung und Lagerung von Kriegsmaterial untersagte. Aber alle diese Maßnahmen waren mehr Ausdruck des Mißtrauens gegenüber den eigenen Alliierten als gegenüber den Deutschen.

Vor diesem Hintergrund war es schockierend für Molotow, daß Byrnes am 15. Mai einen „Vorschlag für die Vorbereitung der Friedensregelung für Deutschland“ auf der Außenministerkonferenz einbrachte, der zwar vorrangig auf die Herstellung der wirtschaftlichen und der Verwaltungseinheit (inkl. der Grenzfragen) abzielte, aber das in

79 FRUS, 1945/III, S. 853-56.

80 Schukow, Erinnerungen, S. 643 ff. DBPO, I, 2, S. 338 f., 408 f., 819.

81 FRUS, 1945/III, S. 859 ff. FRUS, 1945/II, S. 703 ff., $711 \mathrm{ff}$.

82 FRUS, 1946/II, S. 878 f., 889 ff.

83 In die Direktive Nr. 39 vom 2. 10.1946 gingen Teile des Byrnes-Plans ein, ebenso in das Gesetz Nr. 43 vom 29. 12. 1946. Vgl. zur Handhabung der Direktive Nr. 39 unten S. 338 mit Anm. 139 und 140 . 
einer ultimativen Form ${ }^{84}$. Der sowjetische Außenminister fühlte sich düpiert, da er offenbar den Eindruck gewonnen hatte, einem organisierten westlichen Verwirrspiel ausgesetzt zu sein. In seiner Antwort vom 14. Mai auf die Vorstöße von Byrnes und Bevin richtete er das Hauptaugenmerk auf die Ruhrfrage. Ein Friedensvertrag sei für die Sowjetunion völlig ausgeschlossen, ehe nicht ihre Interessen befriedigt waren: industrielle Abrüstung und Kontrolle, Reparation und Transformation. Bizone, ByrnesPlan oder Friedensvertrag, aber auch Internationalisierung nach französischem Modell - alle hatten eines gemeinsam, nämlich daß sie endgültig jeden Einfluß der Sowjetunion auf das Ruhrgebiet ausschlossen ${ }^{85}$. In seiner zweiten Reaktion vom 9. Juli machte Molotow diesen Zusammenhang noch deutlicher: Die bloße Wegnahme der Waffen und die Beseitigung kriegswirtschaftlicher Organisationsstrukturen seien zu wenig, sondern müßten durch eine „industrielle Entwaffnung“ sowie eine „wirkliche alliierte Kontrolle über die deutsche Industrie“ ergänzt werden. Explizit forderte er, diese Maßnahmen mit den politischen Bestimmungen des Potsdamer Abkommens zu verknüpfen, nämlich die Wurzeln des Faschismus durch Land- und Industriereform auszurotten, „besonders in den Westzonen“, und einen demokratischen Neuaufbau zu ermöglichen. Das alles sehe der Byrnes-Plan nicht vor. „So ignoriert der Entwurf die Ziele, die die Alliierten leiteten, als sie die Besatzung Deutschlands beschlossen. Die Präsenz alliierter und [!] sowjetischer Truppen in Deutschland ist durch die folgenden Ziele begründet: erstens die militärische und wirtschaftliche Entwaffnung Deutschlands sicherzustellen und zum Abschluß zu bringen; zweitens die Demokratisierung der Regierung in Deutschland sicherzustellen; und drittens die Reparationslieferungen zu garantieren. Wir sind der Auffassung, daß die Anwesenheit der Besatzungstruppen in Deutschland und die Aufrechterhaltung der Besatzungszonen absolut notwendig sind, solange diese Ziele nicht erreicht sind." Auch wenn die Reparationen nur als dritter Aspekt aufgeführt wurden, so verriet die Ausführlichkeit der folgenden Äußerungen, daß dies der zentrale Punkt, die Grundlage für alles weitere war. Nicht nur sei Clays Reparationsstopp gemäß den Beschlüssen von Yalta und Potsdam „ungesetzlich“, sondern dem Byrnes-Plan wurde vor allem als Versäumnis angekreidet, daß er eine mögliche Beendigung der Besetzung Deutschlands „unabhängig von der Erfüllung der Reparationsleistungen“ vorsah. Die Sowjetunion werde auf Reparationsleistungen in Höhe von $\$ 10 \mathrm{Mrd}$. bestehen. Molotow verhehlte nicht, daß das auch Lieferungen aus der laufenden Produktion meinte ${ }^{86}$.

Seine Ausführungen vom 10. Juli waren nur eine Ergänzung zum Statement vom Vortage, da er auf die westlichen Vorschläge zu Friedensvertrag und Einheit reagieren mußte. Allerdings nutzte er die Chance zu einem Propagandavorstoß, ohne von seinen

${ }^{84}$ FRUS, 1946/II, S. 400 ff. (15. 5. 1946).

85 FRUS, 1946/II, S. 397 ff. PRO, FO 371/55588/C6881 (Prawda, 17.6. 1946). - Obwohl das SMADOrgan, die Tägliche Rundschau, am 19.5. 1946 zum Abschluß der ersten Konferenzphase eine positive Bilanz gezogen hatte („Das Eis wurde gebrochen“), erschien die Sowjetunion nach Eindruck verschiedener Beobachter Mitte Juni zum zweiten Teil in wenig zuversichtlicher Stimmung. FRUS, 1946/II, S. 508 f., 527 f. Der sowjetische Botschafter in Paris nutzte seine pessimistische Lagebeurteilung zu einem kaum verhüllten Angebot an Frankreich, die beiden kontinentalen Mächte Europas müßten enger kooperieren, dabei die „Koinzidenz“ der Auffassungen „in einer gewissen Anzahl von Punkten“ betonend. AMAE, Y 286, Bl. 88.

${ }^{86}$ FRUS, 1946/II, S. 842 ff. Er unterstrich das noch einmal am 11. 7. 1946; ebenda, S. 882 f. Das hatten die Sowjets in der ersten Konferenzphase offiziell noch nicht eingestanden. PRO, FO 943/325 (Bevin, 17.6. 1946, Enclosure). 
inhaltlichen Positionen irgendwelche Abstriche zu machen. Nicht die Zerstörung, sondern die Demokratisierung Deutschlands und seiner Wirtschaft sei das Ziel: „Der Sieg über Deutschland hat mächtige Instrumente in unsere Hände gelegt, um dieses Ziel zu verwirklichen. Es ist unsere Pflicht, diese Mittel bis zum äßersten auszunutzen." Es war nicht ohne inneren Widerspruch, wenn er den Deutschen das Selbstbestimmungsrecht zubilligte, durch ihr Votum die Abtrennung des Ruhrgebiets zu verhindern, aber gleichzeitig den Alliierten das alleinige Recht vorbehielt, das Ruhrgebiet unter internationale Kontrolle zu stellen. Denn prinzipiell strebte jetzt auch die Sowjetunion eine Ausweitung der Produktion unter alliierter Kontrolle an, um die Reparationslieferungen sicherzustellen. Erst in einem zweiten Schritt wandte sich Molotow der Frage eines Friedensvertrags zu: Voraussetzung dafür sei, wie er wiederholt betonte, nicht nur die Bildung einer „ausreichend demokratischen“ Regierung, sondern vor allem deren Bereitschaft und Fähigkeit, „alle ihre Verpflichtungen gegenüber den Alliierten zu erfüllen, einschließlich und ganz besonders im Hinblick auf die Reparationslieferungen an die Alliierten“. Es werde eine „Reihe von Jahren“ in Anspruch nehmen zu überprüfen, wen diese Regierung repräsentiere und ob sie vertrauenswürdig sei - was an sich die Erwartung einer weitgehend kommunistisch kontrollierten Regierung ausschlo ${ }^{87}$. Die Sowjetunion sah sich in der Defensive ${ }^{88}$. Da die Westmächte sich mit Gründung der Bizone von ihrem Veto unabhängig zu machen begannen, da mit der Bizone ein Weststaat und eine Revision des Industrieniveauplans drohten ${ }^{89}$, glaubte die Sowjetunion ihre alten Befürchtungen vor einem „Westblock“ bestätigt - und das deutlich vor dem Marshall-Plan. Eine Einigungschance wurde offenbar nicht mehr gesehen: Zum einen war die Sowjetunion entschlossen, die „antifaschistische" Strukturreform in ihrem Einflußbereich konsequent durchzuführen und dazu die zonale Besatzungsverwaltung aufrechtzuerhalten. Zum zweiten unterstrich der Vorschlag, die Entmilitarisierung von 25 auf 40 Jahre auszudehnen, daß sich die Sowjetunion auf eine langfristige Besatzungsdauer einrichtete. Eine solche wurde zum dritten unumgänglich, wenn sie ihre Reparationsansprüche aus der laufenden Produktion nur der SBZ decken mußte ${ }^{90}$. Überzeugt, daß die Teilung Deutschlands faktisch

87 FRUS, 1946/II, S. 869 ff. Zum sowjetischen Mißtrauen selbst gegenüber einer kommunistischen deutschen Regierung vgl. AMAE, Y 286, Bl. $174 \mathrm{ff}$.

88 Bemerkenswert ist der zeitliche Zusammenhang mit dem SMAD-Befehl Nr. 0212 vom 30. 7., erweitert durch den Beschluß vom 30.10. 1946, mit dem Aufbau einer Zentralverwaltung des Inneren die Zentralisierung und Kasernierung der Polizei in der SBZ einzuleiten. „Durch die gesamte politische Entwicklung Deutschlands ist es absolut notwendig, eine Schutzpolizei zu schaffen. Wir wissen nicht“, das waren allerdings andere Töne, „was morgen in Bayern und anderen reaktionären Gebieten erforderlich [!] wird. Dann braucht man schon eine Schutzpolizei.“ Zitiert nach: Laufer, Ursprünge, S. 158. Im September 1946 äußerte ein sowjetischer Vertreter gegenüber einem französischen Gesprächspartner, die Angelsachsen wollten Deutschland gegen Rußland aufbauen. Seit dem Tod Roosevelts seien in den USA antisowjetische Elemente ans Ruder gekommen. Die Sowjetunion stelle sich auf einen Konflikt innerhalb der nächsten zehn Jahre ein. AMAE, Y 288, Bl. 13 f. Ein von Sokolowski angeordneter Verteidigungsplan lag am 5.11. 1946 vor, der Maßnahmen gegen einen westlichen Vorstoß nach Berlin vorsah. Otkuda groza, S. $26 \mathrm{ff}$.

89 PRO, FO 371/55844/C8111 (Mark Turner, 13.7. 1946). Der britische Botschafter berichtete am 19. 7. aus Moskau, Molotow sei „sehr zufrieden“ mit den Ergebnissen der Pariser Konferenz; "admittedly the Conference had not got very far over Germany but Germany was a complicated question“; FO 371/55589/C8310. Byrnes behauptete, Berichte zu haben, die Sowjets festigten zwar ihren Zugriff auf Osteuropa und auf die SBZ, seien aber völlig unentschieden über ihre langfristigen deutschlandpolitischen Ziele. FO 800/466/Ger/46/33 (3. 10. 1946).

90 In Yalta hatte die Sowjetunion eine industrielle Kontrolle der Alliierten „beyond the period of the reparations payment" von 10 Jahren gefordert. FRUS, Malta and Yalta, S. 620, 631. Daß die Sowjet- 
längst vollzogen sei $^{91}$, stellte die Sowjetunion noch während der Pariser Konferenz ihre Reparationspolitik in der SBZ buchstäblich über Nacht um².

Mit diesem Kurswechsel legte die Regierung in Moskau ihre Deutschlandpolitik endgültig auf einen Alleingang fest: Je mehr die Möglichkeit zur Abschöpfung der Reparationen auf die SBZ reduziert wurde, desto wichtiger wurde die exklusive Kontrolle der Zone wie der Reparationsbetriebe durch Exterritorialisierung der Sowjet AGs. Auch der letzte Weg, diese Entwicklung doch noch zu verhindern, nämlich die Deutschen und ihr nationaler Einheitswille, mußte sich auf die exklusive Kontrolle der SBZ stützen. Diese war ihr Faustpfand und mußte durch innere Reorganisation konsolidiert und westlichem Zugriff entzogen werden. Aber eine derartige Konsolidierung war angesichts der eigenen Reparationspolitik nur möglich durch die Aufrechterhaltung von (eng zu reglementierenden) Wirtschaftsbeziehungen mit den Westzonen, die möglichst geringe Bindungen enthielten, aber ein Maximum an wirtschaftlichen Vorteilen versprachen. Das bedeutete Trennung von politischer und wirtschaftlicher Einheit, das reduzierte wirtschaftliche Einheit auf das Instrument der interzonalen Kooperation und schloß jeden Zugriff alliierter oder gar deutscher Zentralstellen auf die SBZ aus, um eine Rücknahme der dort realisierten Maßnahmen zur „Demokratisierung“ Deutschlands zu verhindern. Nach der Pariser Konferenz fielen die Entscheidungen im Gleichklang mit denen der Westmächte: Die Sowjetunion rückte im August 1946 im Kontrollrat bei Zentralverwaltungen, Reparationen, Währungsreform und Zentralbankfrage von früheren Kompromißpositionen wieder ab. Die „Obstruktion"93 der Sowjetunion offenbarte deren Unfähigkeit, Kompromißlösungen im Kontrollrat herbeizuführen, an denen sie selbst ein ernsthaftes Interesse hatte, die aber eine gewisse Öffnung der SBZ erfordert hätten. Die Sowjetunion war deutschlandpolitisch handlungsunfähig geworden.

union die Dauer der Besatzung neu bestimmte, war auch die Einschätzung der Briten. PRO, FO 371/55844/C7229 (Franklin, 11. 7. 1946), C7885 (12. 7. 1946). Vgl. NA, RG 59, 740.00119 Control(Germany)/7-1146 und /7-1646.

91 Laufer, Ursprünge, S. $151 \mathrm{ff}$.

92 Vgl. unten S. 359 f.

93 AMAE, Y 455, Bl. 76. 